\title{
Lecciones de cuatro meta-análisis globales sobre la restauración de la biodiversidad y los servicios ecosistémicos
}

\author{
José M. Rey Benayas ${ }^{12,}$, $;$ Paula Barral ${ }^{3}$ \& Paula Meli² \\ ${ }^{1}$ Dto. de Ciencias de la Vida, Universidad de Alcalá. Alcalá de Henares, España. ${ }^{2}$ Fundación Internacional para la \\ Restauración de Ecosistemas, España. ${ }^{3}$ Instituto Nacional de Tecnología Agropecuaria (INTA), Argentina.
}

\begin{abstract}
RESUMEN. La restauración ecológica se utiliza a menudo para recuperar los niveles de biodiversidad y de servicios ecosistémicos (SE) en ecosistemas degradados. En general, los resultados de la restauración ecológica se pueden estimar como el progreso o la compleción de la recuperación de indicadores de la integridad ecológica en el estado restaurado respecto al estado degradado o de referencia, respectivamente. Presentamos una síntesis de estos resultados según cuatro meta-análisis globales independientes, obtenidos en una amplia gama de ecosistemas, con énfasis en humedales, agroecosistemas y bosques. La restauración ecológica aumentó la biodiversidad y los SE en 58 y 99\%, respectivamente. Sin embargo, estos niveles fueron inferiores a los de los ecosistemas de referencia (-10 y -8\%, respectivamente). La recuperación varió entre tipos de ecosistema. Los humedales restaurados tuvieron niveles más altos de biodiversidad y de SE (19 y 43\%, respectivamente) en relación a los degradados, aunque los niveles de los SE fueron inferiores (-13\%) a los de referencia. La restauración aumentó los niveles de biodiversidad, SE de soporte y SE de regulación de los agroecosistemas en 68,42 y $120 \%$, respectivamente, y los agroecosistemas restaurados tuvieron niveles de biodiversidad y de estos SE similares a los ecosistemas de referencia. En el caso de los bosques, la recuperación fue completa para todos los SE, mientras que la biodiversidad, aunque aumentó $106 \%$ después de la restauración, fue $21 \%$ inferior a los niveles de referencia. Existe un hueco en la literatura científica sobre la evaluación cuantitativa de los SE culturales en ecosistemas restaurados. La recuperación de la biodiversidad y de los SE se correlacionaron de forma positiva en las comparaciones de ecosistemas degradados y restaurados en todos los meta-análisis. Concluimos que la restauración ecológica aumenta de forma notable la biodiversidad y los SE, aunque no se alcanzan los niveles de los ecosistemas de referencia, y que la efectividad depende, en gran medida, del contexto.
\end{abstract}

[Palabras clave: agroecosistemas, bosques, degradación, humedales, recuperación]

Aвstract. Lessons from four global meta-analysis on biodiversity and ecosystem services restoration. Ecological restoration is often carried out to recover biodiversity and ecosystem services (ES) of degraded ecosystems. In general, the outcomes of ecological restoration can be assessed as the recovery progress or the recovery completeness of indicators of ecological integrity in the restored state against the degraded or reference states, respectively. Here we present the results of four global meta-analyses previously published to assess these outcomes in a wide range of ecosystem types, wetlands, agroecosystems, and forests. For all ecosystem types, ecological restoration increased provision of biodiversity and ES by 58 and 59\%, respectively; however, values of both remained lower in restored versus intact reference ecosystems (-10 and $-8 \%$, respectively). Levels of recovery varied among ecosystem types. Restored wetlands showed 19 and $43 \%$ higher levels of biodiversity and ES, respectively, than did degraded wetlands; however, their levels of ES were lower (-13\%) than in reference wetlands. Restoration increased biodiversity and levels of supporting ES and regulating ES by an average of 68,42 , and $120 \%$, respectively, relative to levels in the pre-restoration agroecosystem, and restored agroecosystems showed levels of biodiversity and these ES similar to those of reference ecosystems. Recovery was complete for all ES, whereas biodiversity, although it increased by $106 \%$ after restoration, was $21 \%$ lower than in reference forests. There is gap related to quantitative assessment of cultural ES provided by restored ecosystems in the scientific literature. Biodiversity and ES response ratios positively correlated in comparisons of restored and degraded ecosystems in all individual meta-analysis. We conclude that ecological restoration markedly enhances biodiversity and ES supply, but the attained levels are lower than those in the reference ecosystems and effectiveness is context dependent to a large extent.

[Keywords: agroecosystems, degradation, forests, recovery, wetlands]

Editor asociado: Mastrangelo Matías

$\bowtie$ josem.rey@uah.es
Recibido: 22 de febrero de 2016

Aceptado: 3 de agosto de 2016 


\section{INTRODUCCIÓN}

El Objetivo 15 del Desarrollo Sostenible (Agenda 2030) indicala necesidad de "Proteger, restaurar y promover el uso sostenible de los ecosistemas terrestres, el manejo sostenible de los bosques, combatir la desertificación, detener y revertir la degradación de la tierra y detener la pérdida de biodiversidad" (ONU 2015). Según la Sociedad Internacional de Restauración Ecológica, la restauración ecológica es el proceso mediante el cual se promueve la recuperación de un ecosistema que ha sido degradado, dañado o destruido, generalmente como consecuencia de las actividades humanas (SER 2004). El concepto original de la restauración es la recuperación de la estructura y la funcionalidad de un ecosistema prístino. No obstante, la identificación de ecosistemas de referencia o modelos de los proyectos de restauración suele ser difícil, o la recuperación del sistema original no es factible. Ello se debe a que, por un lado, la intervención humana ha sido tan intensa y prolongada que los ecosistemas de referencia necesariamente deben integrar el componente antrópico. Este es el caso de ecosistemas culturales como los agroecosistemas. Por otro lado, el clima y los suelos han cambiado a lo largo del tiempo y continuarán haciéndolo en el futuro. Por estas razones y según algunos autores, la restauración ecológica "ortodoxa" debe dar paso a una "restauración ecológica 2.0", más flexible y pragmática (Higgs et al. 2014), cuyo objetivo es la optimización de la biodiversidad, los procesos ecológicos y la provisión de servicios ecosistémicos, teniendo en cuenta el marco ecológico, socio-económico y cultural.

Las actuaciones de restauración ecológica están siendo implementadas de una forma creciente, en particular desde que el Convenio para la Diversidad Biológica propuso como meta de su Plan de Acción para el año 2020 la restauración de al menos $15 \%$ de los ecosistemas degradados del mundo (CBD 2012). Los objetivos más ambiciosos a nivel global tienen que ver con la restauración forestal, como son los relacionados con el Reto de Bonn (2011) y la Declaración de los Bosques de la Cumbre sobre el Cambio Climático (ONU 2014), que persiguen la restauración de 150 y de 350 millones de ha de bosques para los años 2020 y 2030, respectivamente. Sin embargo, hasta la fecha se han realizado pocas evaluaciones sistemáticas de los resultados de la restauración ecológica, es decir, en qué medida ésta es capaz de aumentar la biodiversidad y la provisión de servicios ecosistémicos (SE en adelante) o los beneficios que los humanos obtenemos de los ecosistemas (MEA 2005). Del mismo modo, la relación entre la biodiversidad y la provisión de estos SE es aún incierta.

En general, los resultados de la restauración ecológica pueden estimarse como el progreso de la recuperación o la compleción de la recuperación de indicadores de la integridad ecológica en el estado restaurado respecto a los estados degradado o de referencia, respectivamente. Estos resultados dependen de los denominados factores de contexto, es decir, de multitud de factores determinantes tales como el tipo de ecosistema, el clima, la edad de la restauración, la causa de la degradación y el tipo de acciones de restauración, entre otros. Así, cabe esperar resultados más exitosos en los climas más productivos, si el proceso de la restauración ha operado durante un tiempo relativamente largo y si las causas de la degradación pueden eliminarse fácilmente. Por otro lado, otros factores de contexto de la restauración ecológica tales como el resultado de la intervención humana (restauración activa) frente a la no intervención (restauración pasiva) son controvertidos (Moreno-Mateos et al. 2015).

El objetivo de este trabajo es presentar una síntesis de los resultados de cuatro meta-análisis previos e independientes de estudios de restauración de ecosistemas a escala global: Rey Benayas et al. (2009) relacionado con una amplia gama de ecosistemas, Meli et al. (2014) enfocado en humedales, Barral et al. (2015) enfocado en agroecosistemas y Meli et al. (en revisión) enfocado en bosques. Nuestra hipótesis de partida es que la restauración es capaz de aumentar la provisión de biodiversidad y SE. Investigamos tres preguntas concretas: ¿Qué niveles de biodiversidad y SE se recuperan en los ecosistemas degradados respecto a los restaurados ("progreso de la recuperación") y en los ecosistemas restaurados respecto a los de referencia ("compleción de la recuperación")? ¿Dependen los resultados de la restauración de los factores de contexto? Y, finalmente, ¿Cómo es la relación entre la recuperación de la biodiversidad y la de los SE como consecuencia de la restauración? La respuesta a estas preguntas permitirá plantear proyectos de restauración más exitosos. 


\section{Materiales y Métodos}

En total se analizaron 89, 70, 54 y 166 estudios originales del conjunto de ecosistemas, humedales, agroecosistemas y bosques, respectivamente, de los cuales se obtuvieron 526, 2792, 141 y 1804 medidas cuantitativas de biodiversidad y SE, respectivamente, en tres escenarios comparables: el ecosistema degradado, el ecosistema restaurado y el ecosistema de referencia. Estos cuatro metaanálisis son independientes entre ellos, es decir, parten de una revisión bibliográfica con palabras clave ad-hoc diferentes que han dado lugar a bases de datos distintas utilizadas para responder a preguntas y abordar objetivos específicos que no son siempre coincidentes. Así, por ejemplo, sólo el 8\% de los estudios del meta-análisis de bosques de Meli et al. (en revisión) proceden de la base de datos del meta-análisis más inclusivo de Rey Benayas et al. (2009). Los meta-análisis se basaron en el cálculo de los ratios de respuesta, estimados como $\ln ($ Rest/Deg) y $\ln ($ Rest/Ref), siendo Rest, Deg y Ref las medidas de la misma variable o indicador de la integridad ecológica en los ecosistemas restaurados, degradados y de referencia, respectivamente. Las medidas de biodiversidad se clasificaron en grupos taxonómicos amplios e incluyen abundancia, riqueza de especies, índices de heterogeneidad como el de Shannon-Wiener o Simpson y composición de especies, entre otras. Las medidas de SE fueron clasificadas de acuerdo a los tipos establecidos por el MEA (2005): soporte, provisión, regulación y culturales, y son de muy diferente naturaleza. Desafortunadamente existen muy pocas evaluaciones cuantitativas de los SE culturales en la literatura científica, por lo que éstos no pudieron ser analizados. Según la localización de los ecosistemas, éstos se clasificaron en tropicales y templados, habiendo en general un número notablemente mayor de observaciones procedentes de ecosistemas templados que de tropicales. Una característica común de los cuatro meta-análisis es la elevada heterogeneidad de las escalas espacial y temporal de los análisis. El rango y la media del tiempo desde que comenzaron las acciones de restauración fueron de $<5-300$ años y 29.4 años para el conjunto de ecosistemas, 1.5-55 años y 4 años para los humedales, 1-61 años y 10 años para los agroecosistemas y 0.5-300 años y 28.8 años para los bosques.

Los análisis estadísticos se basaron en pruebas de Wilcoxon para comprobar si los ratios de respuesta eran diferentes a 0 , pruebas de Kruskal-Wallis para comprobar diferencias en las respuestas de distintos factores de contexto y correlaciones no paramétricas para comprobar la relación entre los cambios de biodiversidad y los de SE. Los metaanálisis examinaron los posibles efectos de pseudo-repetición, sin que estos supusieran un problema metodológico. Los cuatro metaanálisis no analizaron consistentemente los mismos factores de contexto ecológico debido a diferencias en los objetivos específicos y número de casos diferentes de cada uno de ellos. Los principales factores de contexto comunes fueron el tipo de ecosistema, el clima, el tipo de restauración (activa o pasiva) y el tiempo de recuperación o edad de la restauración. Otros factores de contexto que se analizaron fueron la principal causa de degradación, las acciones de restauración y el diseño experimental de los estudios originales en el caso de los humedales y la estrategia de la restauración (separación de tierra vs. compartición de tierra) (Rey Benayas and Bullock 2012) en el caso de los agroecosistemas. En el caso de los humedales, los factores de contexto se analizaron mediante modelos lineales de efectos mixtos, en los cuales el estudio original fue considerado un factor al azar que agregaba las medidas obtenidas del mismo. En el caso de los bosques, los factores de contexto se analizaron mediante la comparación de modelos probabilísticos basados en el Criterio de Información de Akaike. Pueden consultarse los trabajos originales de Rey Benayas et al. (2009), Meli et al. (2014) y Barral et al. (2015) para conocer todos los detalles sobre los mismos.

\section{Resultados y Discusión}

\section{¿Qué niveles de biodiversidad y servicios ecosistémicos se recuperan?}

De acuerdo con la hipótesis de partida, los resultados delos cuatro meta-análisisindicaron que la restauración ecológica aumentó, en general, los niveles de biodiversidad y los SE; no obstante, estos niveles fueron inferiores a los de los ecosistemas de referencia (Tabla 1). Para el meta-análisis de todos los tipos de ecosistemas, esta regularidad es consistente excepto para los niveles de los SE de provisión, que son similares en los estados degradado, restaurado y referencia (Tabla 1a). Esto puede probablemente deberse al pequeño número de muestras (Rey Benayas et al. 2009) y a que la extracción de materiales (alimentos, fibra, madera) es una causa de 
Tabla 1. Progreso de la recuperación (comparación entre los ecosistemas restaurados y degradados [columnas RD]) y compleción de la recuperación (comparación entre los ecosistemas restaurados y de referencia [columnas RR]) según cuatro meta-análisis globales de restauración de la biodiversidad y los SE. Los números son \%; nótese que, para la compleción de la recuperación, un número positivo indica que ésta es mayor que la recuperación completa o del 100\%. Los números con asterisco indican diferencias significativas en los niveles de biodiversidad o SE de los ecosistemas restaurados y los degradados o los de referencia (esto no aplica para la última fila (e) de promedios y desvío estándar). NA significa no analizados por déficit de datos. Los SE culturales sólo fueron analizados en los humedales, resultando en niveles similares entre los ecosistemas restaurados y los degradados o los de referencia.

Table 1. Recovery progress (comparison of restored and degraded ecosystems [RD columns]) and recovery completion (comparison of restored and reference ecosystems, [RR columns]) after four global meta-analysis of restoration of biodiversity and ecosystem services (ES). The numbers are \%; note that, for recovery completeness, a positive number means that it is larger than complete or $100 \%$ recovery. Numbers with asterisks indicate statistically significant differences in the levels of biodiversity and ES in restored and degraded or reference ecosystems (this does not apply to the last row (e) of means and standard deviations). NA means insufficient data for analyses. Cultural ES were only analyzed in wetlands resulting in similar levels in restored and degraded or reference ecosystems.

\begin{tabular}{lccccccccccc}
\hline \multicolumn{1}{l}{ Tipo de ecosistema } & \multicolumn{1}{c}{ Biodiversidad } & \multicolumn{9}{c}{ Todos los SE } & \multicolumn{9}{c}{ SE de soporte SE de regulación SE de provisión } & Fuente \\
& RD & RR & RD & RR & RD & RR & RD & RR & RD & RR & \\
\hline a. Todos & $38^{*}$ & $-14^{*}$ & $25^{*}$ & $-20^{*}$ & $28^{*}$ & $-18^{*}$ & $20^{*}$ & $-44^{*}$ & -24 & 23 & $\begin{array}{c}\text { Rey Benayas } \\
\text { et al. 2009 }\end{array}$ \\
b. Humedales & $19^{*}$ & -2 & $43^{*}$ & $-13^{*}$ & $40^{*}$ & $-16^{*}$ & $47^{*}$ & $-22^{*}$ & $80^{*}$ & -7 & $\begin{array}{c}\text { Meli et al. } \\
2014\end{array}$ \\
c. Agroecosistemas & $68^{*}$ & -3 & NA & NA & $42^{*}$ & -18 & $120^{*}$ & 22 & NA & NA & $\begin{array}{c}\text { Barral et al. } \\
2015\end{array}$ \\
d. Bosques & $106^{*}$ & $-21^{*}$ & $228^{*}$ & 9 & NA & NA & $131^{*}$ & -5 & NA & NA & $\begin{array}{c}\text { Meli et al. en } \\
\text { revisión }\end{array}$ \\
e. Promedio \pm DE & $58 \pm 38$ & $-10 \pm 9$ & $99 \pm 112$ & $-8 \pm 15$ & $37 \pm 8$ & $-17 \pm 1$ & $79 \pm 54$ & $-12 \pm 28$ & $28 \pm 74$ & $8 \pm 21$ & r
\end{tabular}

degradación de los ecosistemas (Rodríguez et al. 2006). Los humedales restaurados tuvieron niveles de biodiversidad y SE más altos que los degradados; sin embargo, los niveles de biodiversidad fueron similares y los de los SE excepto los de provisión fueron inferiores a los de los humedales de referencia (Tabla 1b) (Meli et al. 2014). Del mismo modo, la restauración aumentó los niveles de biodiversidad y SE de los agroecosistemas, $\mathrm{y}$ los agroecosistemas restaurados tuvieron niveles de biodiversidad y SE similares a los ecosistemas de referencia (Tabla 1c) (Barral et al. 2105). En los bosques, aunque los niveles de biodiversidad aumentaron considerablemente en los ecosistemas restaurados con respecto a los degradados, la recuperación con respecto al ecosistema de referencia resultó incompleta. Por el contrario, la restauración aumentó los niveles de todos los SE hasta su completa recuperación (Tabla 1d) (Meli et al. en revisión).

Así, en respuesta a nuestra primera pregunta, el progreso de la recuperación (la comparación de los ecosistemas degradados con los restaurados) promedió un 58\% para la biodiversidad y un $99 \%$ para el conjunto de los SE en los cuatro meta-análisis realizados (Tabla 1e). Por otro lado, la compleción de la recuperación (la comparación de los ecosistemas de referencia con los restaurados) promedió $-10 \%$ para la biodiversidad y $-8 \%$ para los SE (aunque la biodiversidad se recuperó totalmente en los humedales y en los agroecosistemas, pero no en los bosques). Los SE se recuperaron totalmente en los agroecosistemas y en los bosques atendiendo a las diferencias estadísticas $(P>0.05)$. En consecuencia, podemos afirmar que, en una gran variedad de ecosistemas y a diferentes escalas espaciales y temporales, la restauración ecológica es relativamente exitosa.

\section{¿Dependen los resultados de la restauración de los factores de contexto?}

Encontramos que los resultados de la restauración ecológica dependieron de los siguientes factores de contexto. 1) Para el conjunto de los ecosistemas, el tipo de ecosistema (terrestre vs. acuático) en combinación con el clima (templado vs. tropical), el progreso de la recuperación de la biodiversidad y los SE fue mayor en los ecosistemas acuáticos que en los terrestres, mientras que la compleción de la recuperación fue mayor en los ecosistemas templados que en los tropicales (Rey Benayas et al. 2009). 2) En los humedales, el progreso de la recuperación dependió de la principal causa de degradación, las acciones de restauración, el diseño experimental de los distintos estudios originales y el tipo de humedal (Meli et al. 2014). La restauración ecológica mejoró los niveles de biodiversidad y SE de los humedales afectados por las diferentes causas de degradación excepto por la invasión de especies exóticas. Las acciones de restauración más exitosas fueron la creación de hábitats, 
las enmiendas edáficas y la revegetación. Los experimentos pareados resultaron en mayores efectos que los experimentos antes-después y los control-impacto. Los humedales salinos fueron los que elevaron más sus niveles de biodiversidad y de SE, y los ríos fueron los que menos como consecuencia de la restauración. Mientras tanto, la compleción de la restauración de los humedales dependió básicamente del tipo de acción de restauración. En general, la restauración promueve una recuperación completa, excepto cuando se realiza una acción conjunta de revegetación y mejora del suelo, restauración pasiva, manipulación de la heterogeneidad estructural o de la dinámica hidrológica (Meli et al. 2014). 3) En los agroecosistemas, la estrategia de la restauración (separación de tierra vs. compartición de tierra) sólo afectó de forma significativa al progreso de la recuperación de los SE, siendo mayor en los estudios relacionados con la primera estrategia, mientras que el tipo de restauración (pasiva vs. activa) no afectó a los resultados de la restauración (Barral et al. 2015). 4) En los bosques, la compleción de la recuperación dependió principalmente del tipo de medida (i.e., la abundancia, la diversidad y la función) y del tiempo de recuperación (Meli et al. en revisión).

La recuperación de la función y la biodiversidad de los bosques restaurados de forma activa aumentaron a lo largo del tiempo, pero el tiempo de recuperación no afectó a los resultados de la restauración en humedales y agroecosistemas, y no fue probada para el conjunto de ecosistemas. Por consiguiente, en respuesta a la segunda pregunta, los resultados de la restauración ecológica dependieron de distintos factores de contexto ecológico pero estos no fueron consistentes en los diferentes meta-análisis.

\section{¿Cómo es la relación entre la recuperación de la biodiversidad y la recuperación de los SE como consecuencia de la restauración?}

En respuesta a la tercera pregunta, el progreso de la recuperación de la biodiversidad y los SE se correlacionó de forma positiva en el conjunto de ecosistemas, humedales, agroecosistemas y bosques. La compleción de la recuperación de la biodiversidad y los SE también se correlacionó de forma positiva en el conjunto de ecosistemas, humedales y bosques, pero no se analizó en los agroecosistemas. Tanto en el conjunto de ecosistemas como en humedales y bosques, la relación entre el progreso de la recuperación de la biodiversidad y los SE fue más fuerte que la relación para la compleción de la recuperación. Estas relaciones indican, en general, una doble ganancia de recuperación de niveles de biodiversidad y SE a lo largo del proceso de la restauración y, aparentemente, una saturación de la función ecológica con niveles elevados de biodiversidad (Rey Benayas et al. 2009; Cardinale et al. 2011).

\section{Representatividad de los meta-análisis}

El propósito de cualquier meta-análisis es sistematizar las tendencias generales de unos datos obtenidos por distintos estudios primarios que son inherentemente complejos (Harrison 2011). Este trabajo ha producido una sinopsis de cuatro metaanálisis independientes a escala global $y$, en consecuencia, los consideramos representativos de los resultados de la restauración ecológica en los niveles de biodiversidad y servicios ecosistémicos, excepto los culturales, alcanzados en una amplia gama de ecosistemas y a diferentes escalas espaciales y temporales. Los metaanálisis de los humedales y los agroecosistemas están sesgados hacia las zonas templadas del planeta por una deficiencia de estudios primarios en áreas tropicales, lo que debe tenerse en cuenta a la hora de interpretar sus resultados. Ningún meta-análisis de los presentados estuvo afectado por problemas de pseudo-repetición. Una prueba cuantitativa del sesgo de la selección de casos requiere medidas del número de datos de cada estudio primario y de la varianza asociada, los cuales no estaban disponibles en todas nuestras bases de datos. No obstante, consideramos que el sesgo de publicaciones de nuestros meta-análisis es bajo por dos razones. La primera es que el propio interés en determinar si las restauraciones son exitosas o no hace improbable que no se reporten las poco exitosas. La segunda es que los SE que analizamos no se reportaron como tales en la mayor parte de los estudios primarios sino como medidas no sesgadas de procesos o funciones y nuestra re-interpretación de las mismas diluiría cualquier posible sesgo (Rey Benayas et al. 2009).

\section{Conclusiones}

Concluimos que la restauración ecológica en una variedad de ecosistemas y de escalas tanto espaciales como temporales es notablemente efectiva para aumentar 
los niveles de biodiversidad y de SE (58 y $99 \%$, respectivamente) (Tabla 1e), aunque esta efectividad depende en gran medida de los factores de contexto. No obstante enfatizamos que, en analogía al "es mejor prevenir que curar" en referencia a la salud, también es preferible conservar ecosistemas con elevada integridad ecológica a tener que restaurarlos, máxime teniendo en cuenta que frecuentemente los ecosistemas restaurados no alcanzan los niveles de biodiversidad y SE (-10 y $-8 \%$, respectivamente) (Tabla 1e) de los ecosistemas naturales de referencia y que, además, la recuperación de estos niveles puede ser lenta. Las acciones de restauración del capital natural deben ser un motor de una nueva economía de mercado y una fuente de empleos verdes, lo que significa un incentivo para los políticos y ciudadanos en general para emprender proyectos de restauración de una forma generalizada.

Agradecimientos.Los autores agradecen principalmente los comentarios de los revisores de los tres meta-análisis originales publicados, dos revisores y el editor Matías Mastrangelo de la revista Ecología Austral y los organismos que los financiaron (CGL201018312 de CICYT de España, S2009AMB1783-REMEDINAL-2 y S2013/MAE-2719 REMEDINAL-3 de la Comunidad de Madrid y PICT 2008-0020 de FONCyT de Argentina). JMRB y PM realizaron el meta-análisis de restauración forestal con colegas del grupo de trabajo "A Restoration Synthesis" del SESYNC (https:/ / www.sesync.org/project/ restoration-synthesis), con financiación de la National Science Foundation DBI-1052875, el German Helmholtz Centre for Environmental Research - UFZ Leipzig (Research Program 'Terrestrial Environments') y el sDiv, the Synthesis Centre of the German Centre for Integrative Biodiversity Research (iDiv) HalleJena-Leipzig (German Research Foundation DFG FZT 118), además de por un proyecto del programa KNOW-FOR de la UICN a la FIRE gracias al Department for International Development (DFID).

\section{REFERENCIAS}

Barral, P., J. M. Rey Benayas, P. Meli, and N. Maceira. 2015. Quantifying the impacts of ecological restoration on biodiversity and ecosystem services in agroecosystems: a global meta-analysis. Agr Ecosyst Environ 202:223-231.

Cardinale, B. J., K. L. Matulich, D. U. Hooper, J. E. Byrnes, E. Duffy, et al. 2011. The functional role of producer diversity in ecosystems. Am J Bot 98:572-592.

CBD. 2012. Strategic Plan for Biodiversity 2011-2020 and the Aichi Targets. URL: www. cbd.int/doc/strategic-plan/ 2011-2020/Aichi-Targets-EN.pdf.

Bonn Challenge. 2011. URL: www.bonnchallenge.org/content/challenge.

Harrison, F. 2011. Getting started with meta-analysis. Method Ecol Evol 2:1-10.

Higgs, E., D. A. Falk, A. Guerrini, M. Hall, J. Harris, R. J. Hobbs, and W. Throop. 2014. The changing role of history in restoration ecology. Fron Ecol Environ 12:499-506.

Meli, P., J. M. Rey Benayas, P. Balvanera, and M. Martínez-Ramos. 2014. Restoration enhances wetland biodiversity and ecosystem service supply, but results are context-dependent. PLOS ONE 9(4): e93507.

Meli, P., K. D. Holl, J. M. Rey Benayas, H. P. Jones, P. Jones, et al. Do forests recover after logging, agriculture, and mining? PLOS ONE (under review).

MEA (Millennium Ecosystem Assessment). 2005. Ecosystems and Human Well-being: Synthesis. Island Press, Washington, DC.

Moreno-Mateos, D., P. Meli, M. I. Vara-Rodríguez, and J. Aronson. 2015. Ecosystem response to interventions: lessons from restored and created wetland ecosystems. J Appl Ecol 52:1528-1537.

ONU. 2014. Declaración de los Bosques de la Cumbre sobre el Cambio Climático. URL: http://newsroom.unfccc.int/ es/bienvenida/cumbre-del-clima-resumen-principales-resultados/.

ONU. 2015. Objetivos de Desarrollo Sostenible. URL: www.un.org/sustainabledevelopment/es/objetivos-de-desarrollosostenible/.

Rey Benayas, J. M., A. C. Newton, A. Díaz, and J. M. Bullock. 2009. Enhancement of biodiversity and ecosystem services by ecological restoration: a meta-analysis. Science 325:1121-1124.

Rey Benayas, J. M., and J. M Bullock. 2012. Restoration of biodiversity and ecosystem services on agricultural land. Ecosystems 15:883-889.

Rodríguez, J. P., J. R. Beard, M. E. Bennett, G. Cumming, S. Cork, J. Agard, et al. 2006. Trade-offs across space, time, and ecosystem services. Ecol Soc 11(1):28.

SER. 2004. SER International Primer on Ecological Restoration. URL: www.ser.org/resources/resources-detail-view/ ser-international-primer-on-ecological-restoration. 